\title{
On Invoking Third Parties in Vietnamese Medical Communication
}

\author{
Huong Thi Linh Nguyen \\ School of Linguistics, Adult and Specialist Education, Faculty of Business, Education, Law and Arts, University of \\ Southern Queensland, Toowoomba, QLD 4350, Australia \\ Gavin Austin \\ School of Linguistics, Adult and Specialist Education, Faculty of Business, Education, Law and Arts, University of \\ Southern Queensland, Toowoomba, QLD 4350, Australia
}

\begin{abstract}
This study is concerned with the practice of invoking third parties among doctors and patients in Vietnamese medical consultations. These third parties are relatives of the patient who are also medical professionals. We show that doctors invoke relatives-plus-medical professionals in order to elicit information from patients, while patients adopt this practice in order to circumvent a troublesome administrative requirement; obtain a preferred form of treatment; receive a health-related service from the hospital, while also diminishing accountability for making this request in case it turns out to be irregular; give reasons for selecting the current hospital; or challenge the doctor's expertise. Another possible motive is to receive special attention from the treating doctor. We suggest that doctors and patients are particularly inclined to invoke relatives-plus-medical professionals as third parties because of two social forces within Vietnamese culture: collectivism and social status. We also adduce evidence that, as a determinant of the patient's future treatment for their problem, their familial relationship with the third party overrides this person's status as a medical professional in this cultural context. More broadly, our findings indicate that medical communication is not invariant across cultures, but can be shaped by culture-specific forces.
\end{abstract}

Index Terms—medical communication, third parties, cultural context, Vietnam, Conversation Analysis

\section{INTRODUCTION}

One feature of doctor-patient communication that has been noted in the research literature is the practice of invoking third parties (Bergmann, 1992; Gill \& Maynard, 2006; Heritage \& Robinson, 2006). These individuals have included medical professionals (e.g., general practitioners), professionals from other fields (e.g., social workers or the police), and non-professionals (e.g., family members or friends). In addition, the practice itself has been found to occur at various stages of the consultation. Yet, despite this interest on the part of investigators in this field, it would be fair to say that a dedicated and in-depth examination of the practice of invoking third parties among doctors and patients is still called for. The chief aim of the present study is to address this research deficit.

Moreover, our investigation is situated in the Vietnamese cultural context. Within research dealing with medical discourse generally, this context has so far garnered relatively little attention (for details, see H. T. L. Nguyen et al., 2018). In the current study, we aim to augment the relatively meagre stock of research on Vietnamese doctor-patient discourse in general, and make scholarly coverage of this type of discourse more representative in cross-cultural terms as a result.

Quite apart from this lack of research, the Vietnamese cultural context seems a promising one in which to investigate the practice of invoking third parties. There are two main reasons for this. To begin with, Vietnamese society is collectivist (Bao Dat, ${ }^{1}$ 2001; Hofstede, 2001), a consequence of an agrarian lifestyle which emphasises the importance of interdependence among people in cultivating food (V. Q. Tran, To, Nguyen, Lam \& Tran, 1998). The influence of this collectivism is particularly keenly felt within family relationships (Kádár \& Mills, 2011; Thompson, 1965), especially when important decisions need to be made (Appel, 2013; Kim, Klingle, Sharkey, Park, Smith, \& Cai, 2000; T. X. Nguyen, 1994).

The second reason is that Vietnamese society has a deeply-ingrained hierarchical structure (Edwards \& Phan, 2013) which will likely reveal itself in doctor-patient discourse as well. And indeed this expectation is borne out in the extant literature. According to Pham (2014), doctors in Vietnam often presuppose that patients meet them to address biomedical concerns only, and formulate their information-seeking moves accordingly. In studies on the patient's perspective, the main finding has been that these participants tend to adopt an inhibited, compliant attitude towards those who are charged with treating them. Fancher et al. (2010) found that the patients in their study rarely expressed their concerns directly unless asked. In G. Nguyen et al. (2007), even though most of the patients reported being

\footnotetext{
${ }^{1}$ This author's name consists of a middle name plus first name only (i.e., there is no surname). Accordingly, we have cited each of these names in full.
} 
dissatisfied with their communication with their doctors in certain respects, they still gave credence to what their doctors told them about their health. Almost all of the patients in K. Tran (2009) claimed to have only spoken when invited to do so by the doctor. Tran adds that this behavior was motivated by a desire to minimise any imposition on the doctor's autonomy, avoid hurting the doctor's feelings, or reduce the likelihood of any negative evaluation of the patient's low English proficiency.

These two characteristics of Vietnamese society have led us to select a particular type of third party for close scrutiny in the present study. Our view is that the collectivist aspect of this culture especially favours the invocation of third parties who are relatives of the patient. At the same time, the hierarchical structure of Vietnamese society has its own implications for this practice. A third-party medical professional not only enjoys relatively high social status but is also an 'insider' working in the same field (and perhaps even in the same hospital). In this light, third-party medical professionals might be expected to exert more influence over the outcome of medical consultations than high-status third parties from other walks of life. Accordingly, each of the third parties included in this study is a relative of the patient who also happens to be a medical professional ${ }^{2}$ (cf. Bergmann, 1992; Gill \& Maynard, 2006; Heritage \& Robinson, 2006).

A noteworthy feature of previous work on doctor-patient discourse in the Vietnamese cultural context in general is that, unlike Bergmann (1992), Gill and Maynard (2006) and Heritage and Robinson (2006), no study has adopted Conversation Analysis as its framework (for a comprehensive overview of how $\mathrm{CA}^{3}$ has been applied to medical communication, see Gill \& Roberts, 2013). In this respect, the current study fills a significant methodological gap.

\section{THIRD PARTIES In MEDiCAL COMMUNiCATION}

Studies which deal, at least in part, with the practice of invoking third parties among doctors and patients have been quite varied in certain respects. In one study, this practice is employed by health professionals. Bergmann (1992) discovered that psychiatrists invoke third parties within discreetly exploring utterances in intake interviews as a strategy for eliciting information from prospective patients about their ${ }^{4}$ mental state. Along with the patient's behavior in the interview, this information is used by the medical professional to decide if the patient should be hospitalised. In Extract 1 , a psychiatrist is talking to a married couple, and must determine if the wife should be committed. He has just concluded a telephone call with the referring doctor (data from p. 138).

Extract 1

1 Dr.F (I just) got the information, (0.8)

2 (that you're) not doing so well.

3 Ms. B Yea::h well that is [the opinion

4 Dr.F [Is that correct?

5 Ms. B of Doctor Hollmann.

In his initiating move in lines 1-2, the psychiatrist indicates that his knowledge of the patient's state comes from another source ('(I just) got the information'), and is therefore not necessarily accurate. Bergmann suggests that, in framing his knowledge in these terms, the psychiatrist is inviting the patient to supply an authoritative account of her health condition. The provenance of his knowledge is then identified in Ms. B's response in line 3.

Other studies are concerned instead with the invocation of third parties by patients. Two motives for engaging in this practice have been identified. In Gill and Maynard (2006; see also Gill, 1998), it is used when the patient embeds an explanation for their symptoms within a frank question which, in addition to dealing with sensitive information, limits the doctor's possible range of responses. Gill and Maynard point out that, each time this type of question is put to the doctor in their data, this occurs during a phase of the consultation where they are not gathering information. Consider Extract 2. Notice that Ms. N mitigates her question about the cause of her depression by invoking her health aide, and also that this question does not receive an edifying response (data from p. 127).

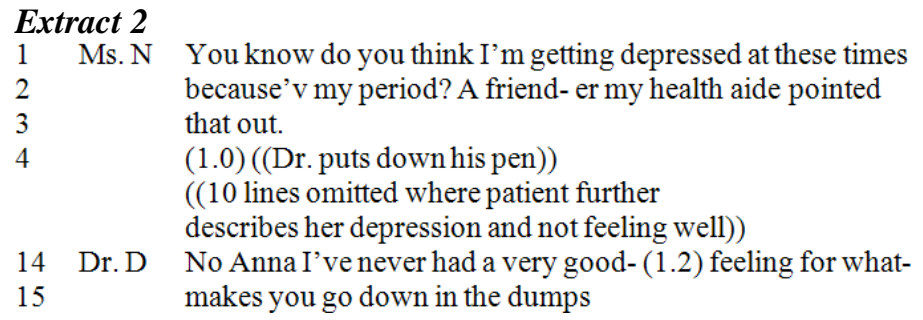

In Heritage and Robinson (2006), patients invoke third parties in order to present their health concerns as reasonable and well-founded, while simultaneously also diffusing responsibility for taking the step of seeking treatment. This is exemplified in Extract 3. In the past, this patient has received treatment for a carcinoma on the back of her neck, and she

\footnotetext{
${ }^{2}$ Note that we do not refer to the third party as 'a medical professional who also happens to be a relative of the patient.' In our analysis of Extract 6 later on, we will see that the ordering used in the main text more accurately reflects the comparative importance of these two at tributes of the third party in determining the outcome of the consultation.

${ }^{3}$ The following abbreviations are used in this article: CA - Conversation Analysis; CLA - classifier; COP - copula; D - doctor, HON - honorific; INT - interrogative; P - patient, PRT - particle; PST - past tense; RM - relative-plus-medical professional.

${ }^{4}$ Throughout this paper, we use the gender-neutral pronoun 'they/their etc.' if the referent's gender is unspecified.
} 
has just found a suspicious bump (referred to as a "mole" in line 7) at, or near, the same location as before (data from p. 59).

\section{Extract 3}

$1 \quad \mathrm{P} \quad$ I'm here on fal[se pre- pretenses. $<$ I think.

2 D

3 D

$4 \quad \mathrm{P}$

((Five lines omitted))

$6 \quad$ P I asked my husband yesterday 'cause I could feel: (0.8) (cause)

$7 \quad$ I: could feel this li'l mo:le coming. An:d: $u h(0.5)($ he) (.) I:

$8 \quad$ hh thought I better letchya know-<uh well I asked my husband' $\mathrm{f}$

$9 \quad$ it was in the same place you took off thuh $(0.5)$ othee $(\mathrm{mm})$

The patient expresses uncertainty about the legitimacy of her concerns overtly in line 1 . This is followed up in lines 6 and 8 , where she diminishes her own accountability for seeking medical attention by invoking her husband.

The studies cited above have three key limitations. In the first place, there is the fundamental problem that the invocation of third parties in doctor-patient communication has only been sparsely investigated. Indeed, to the best of our knowledge, the only published study to have examined its use by medical professionals of any sort (in contrast to patients) has been Bergmann (1992).

Second, this dearth of scholarly attention is exacerbated by the fact that, even when researchers have shown interest in this phenomenon, it has been afforded only subsidiary importance within studies concerned with other issues. Bergmann's overriding objective is to show that, instead of treating a discreetly exploring utterance as a gambit which invites the prospective patient to talk about their problems in their own words, the patient may instead regard it as a bid to unearth evidence of conduct that is, in some sense, improper. Whether or not the utterance invokes a third party is beside the point. Gill and Maynard (2006) analyse how the patient puts forward an explanation for their medical problems during the information-gathering phase of the consultation, and how the doctor responds to this potential disruption, while Heritage and Robinson (2006) explore the ways in which the patient justifies their decision to see a doctor. Within each of these last two studies, the invocation of a third party is merely one of several means of achieving a particular end.

Lastly, note that the focus of Bergmann's (1992) study is psychiatric care. This is a concern if we allow for the possibility that the behavior of this group may not be representative of medical professionals more generally: while the information that psychiatrists need to elicit is, by its very nature, highly sensitive, this is not always true of general practitioners. In this light, the invocation of third parties by doctors in particular seems even more deserving of further investigation.

In the present study, we seek to address these limitations of previous work in this specific area.

\section{Methodology}

The data for this study comes from 66 primary-care visits at two public hospitals in Vietnam. ${ }^{5}$ The visits were recorded between June and August 2016. Ethical clearance for the study was granted by the University of Southern Queensland. Fifteen doctors and 66 patients took part in the study. The data was collected from audio recordings, and all of it was transcribed in the original Vietnamese by the first author using ELAN software. The extracts included in the present paper were then translated into English by both authors. Finally, all participants were de-identified using pseudonyms. The CA transcription notation used in this study is based on the one found in Jefferson (2004), except that one symbol (i.e., the hash (\#) sign) has been added. The participants in this project often produced certain words so quickly that they were almost inaudible (e.g., the word không ('no') in Tôi không biết ('I don't know'), when this formulaic chunk was used). In a case like this, the swallowed utterance or part thereof is enclosed within hashes (e.g., Tôi \#không\# biết) in the data extracts.

\section{RESULTS AND ANALYSIS}

In this section, we look at the invocation of RMs by doctors and then by patients. All instances of RM invocation by the patients occurred when the patient expanded their response to an elicitation by the doctor. This section includes every instance that occurred in the 66 visits.

\section{Doctors invoking RMs}

In Extract 4, doctor Chu invokes an RM working in his hospital in order to elicit information from patient Sang. Sang has pain and numbness in her legs. ${ }^{6}$

\section{Extract 4}

\footnotetext{
${ }^{5}$ In the present paper, we do not discuss all 66 visits, but limit our coverage to the ones which contain examples of the phenomenon under investigation.

${ }^{6}$ This exchange happened during the history-taking and physical examination stage of the consultation. The discourse which occurred immediately before this exchange was concerned with the problem with Sang's legs.
} 


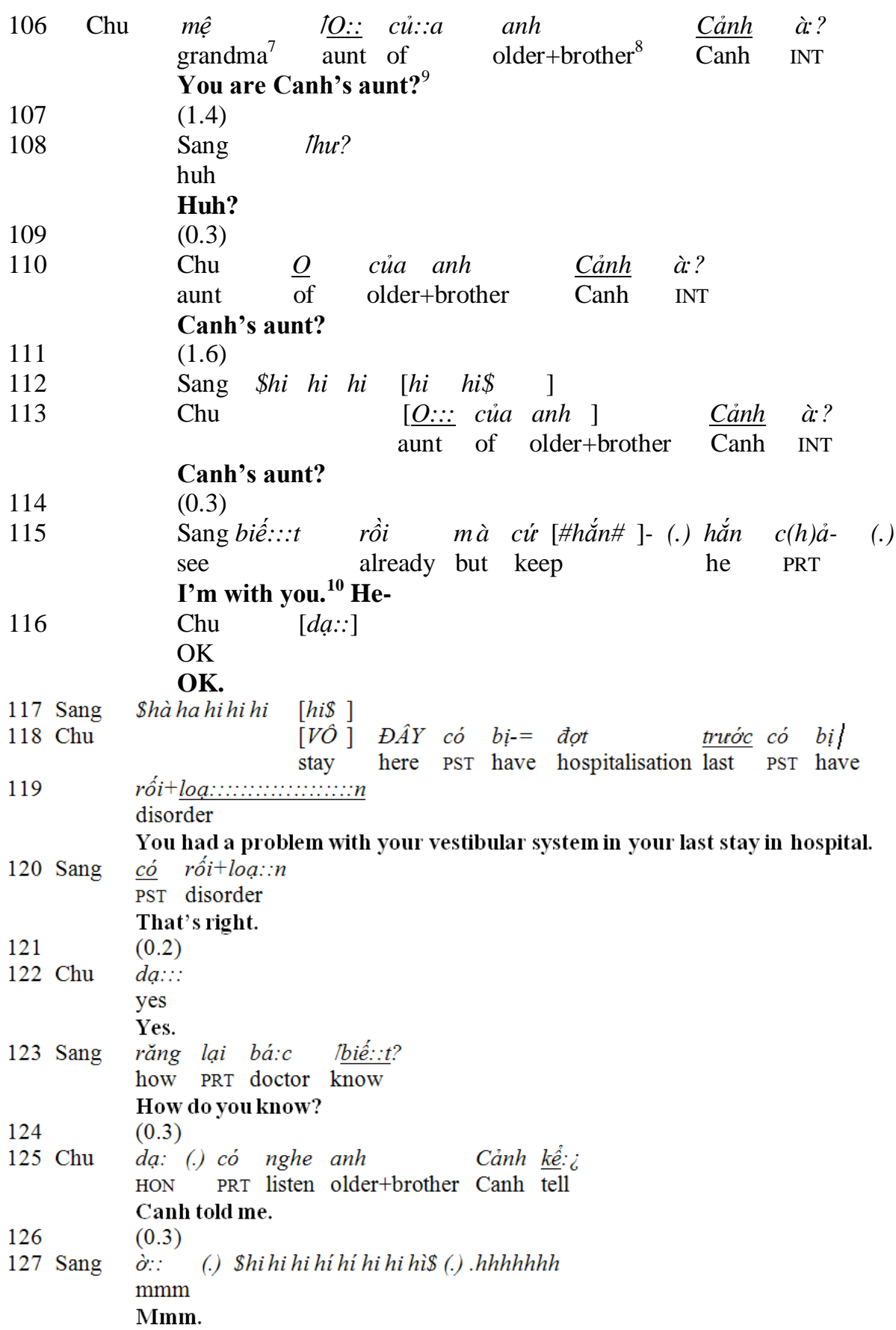

After a lapse of 6.1 seconds (data not shown), doctor Chu abruptly moves to the topic of Sang's nephew, a doctor named Canh. Chu poses a question ending with the particle à: (line 106) polarised in a positive direction to invite a preferred next action (Pomerantz, 1984). After a delay of 1.4 seconds (line 107), Sang's use of an open-class form (Drew, 1997), Thu? ('Huh?'; line 108), signals that she is having difficulty understanding Chu's talk. This turn treats Chu's question as an other-initiated repair (Schegloff, Jefferson, \& Sacks, 1977) which suspends the progressivity of the sequence. It is not an answer to Chu's question, but starts off a new question-answer sequence. Chu thus reconstructs his turn as a repair (line 110). After a 1.6-second silence (line 111), Sang laughs instead of answering Chu's question (line 112). This laughter communicates that Sang registers Chu's request as delicate, thus displaying her

\footnotetext{
${ }^{7}$ The hierarchical organisation of Vietnamese society is reflected in a large number of kinship terms used for addressing and referring to others (for more information, see H. T. L. Nguyen et al., 2018).

${ }^{8}$ We use a plus $(+)$ sign to join together two or more words in the Vietnamese transcription. The other options conventionally u sed for this purpose (e.g., a period or a hyphen) are unsuitable, as both these symbols have values within the CA transcription system. For consistency, we use the same symbol for this purpose in the interlinear morpheme gloss as well.

${ }^{9}$ In morphosyntactic terms (including the use of ellipsis), Vietnamese and English diverge considerably (H. D. Nguyen, 2009). Our priority in the translations is to strike a balance between the naturalness of the English on one hand and faithfulness to the original on the original. For the sake of clarity, we also occasionally add some information that is left implicit in the original.

${ }^{10}$ In other words, the patient is able to follow what the doctor is telling her.
} 
reluctance to disclose this information to Chu (Haakana, 2001). This triggers Chu's repeat of his own question for the second time (line 113). At this point, Sang acknowledges her receipt of his question (line 115); however, this conforms to neither the question's action nor its topical agenda (Raymond, 2003), as her response does not contain 'yes' or 'no', or answer Chu's question. Having received Sang's non-answer response (Enfield, Stivers, \& Levinson, 2010), Chu

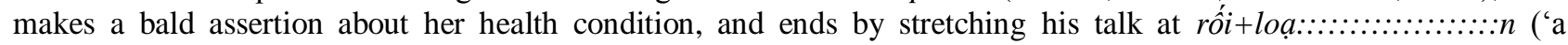
problem with your vestibular system'; lines 118-119). Sang then confirms Chu's presupposition (line 120), but expresses uncertainty about how Chu could have obtained this information (line 123). In response, Chu attributes this information to Canh (line 125).

In this extract, doctor Chu invokes an RM (i.e., doctor Canh; line 106) as a fishing ${ }^{11}$ device to elicit information from Sang. Once he has elicited this information, Chu is able to convince her of the veracity of this information as well (line 125), since it came from an RM.

\section{Patients invoking RMs}

In the first extract to be considered in this section, patient Cam invokes her daughter-in-law, Linh, who is a doctor in a large hospital in the community, in explaining why she has not brought the necessary medical documentation to the consultation. Cam has had back and leg pain for four months, and has sought treatment for these difficulties at another hospital before. Extract 5 takes place right after doctor Hung has asked Cam to show him any medical papers related to her previous treatment.

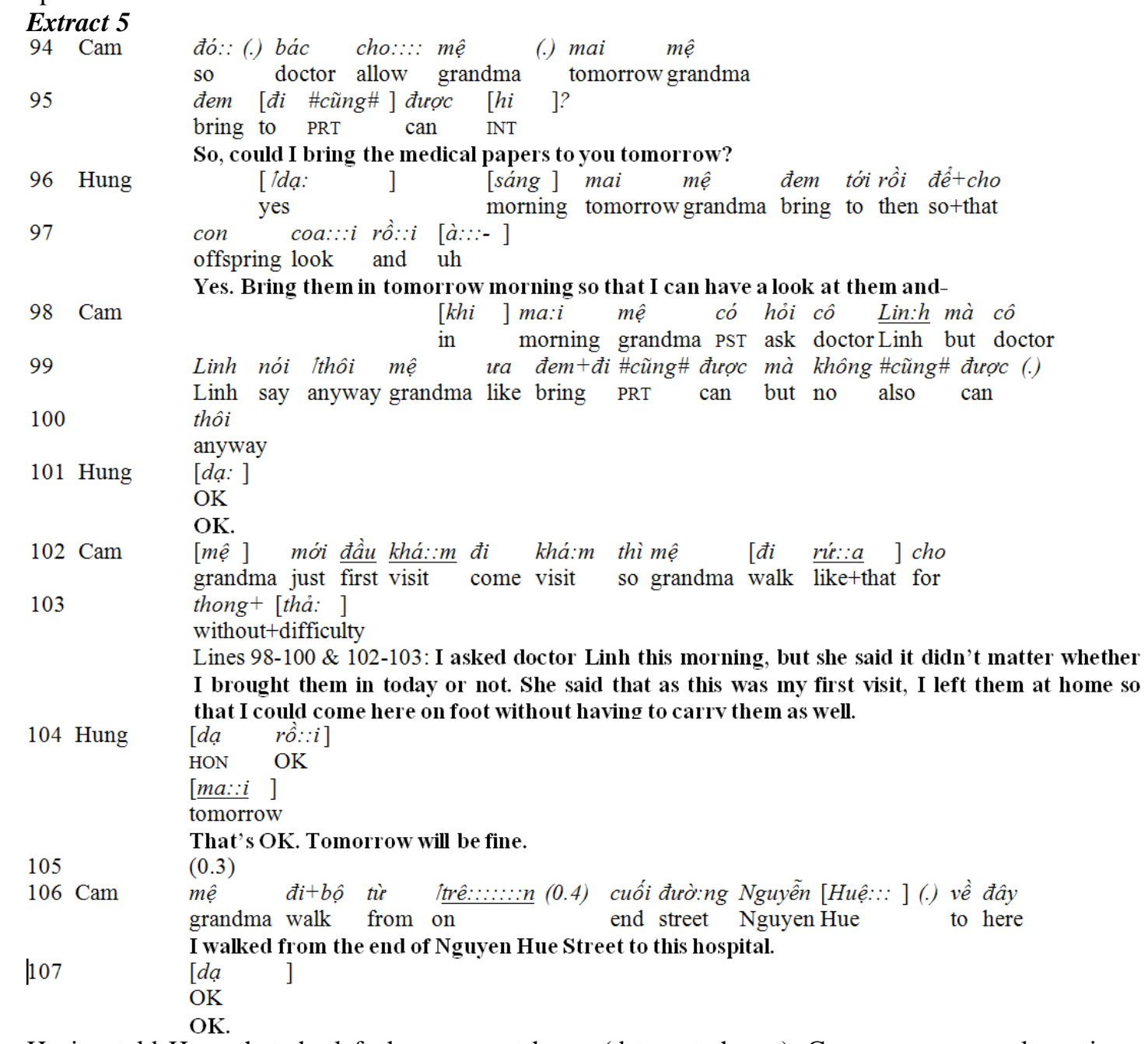

Having told Hung that she left the papers at home (data not shown), Cam proposes an alternative solution to this problem at lines 94-95. Hung's uptake registers her proposal as acceptable (lines 96-97). In this turn, he only states the reason why the papers are needed, without making any complaint about Cam's actions. However, Cam's next turn reveals that she has interpreted Hung's utterance as a complaint (Sidnell, 2012). In khi ma:i mệ có hỏi cô Lin:h ('I asked doctor Linh this morning'), Cam implies that she is not at fault for leaving the papers at home: she has merely acted on the advice of her daughter-in-law, who is a medical expert. In this account (lines 98-100 and 102-103), Cam reports the advice of Linh, mê đi rú:::a cho thong thả: ('I could come here on foot without having to carry them as well'), to imply that she has difficulty walking. On receiving Hung's acceptance of her suggestion (line 104), Cam then expands her talk

\footnotetext{
11 'Fishing' is a term coined by Pomerantz (1980) to describe a technique that the doctor uses to collect information when they have limited knowledge of the patient's health condition ahead of the consultation.
} 
to offer further justification for her actions (line 106). She disloses the location of her home, and through a self-initiated repair, replaces Itrê.:.:.:n ('on') with cuối ('end') to emphasise the long distance from her home to this hospital. The implication is that, while walking itself is difficult enough for a patient with leg pain, carrying papers such a long distance will be especially so.

On their own, Cam's physical limitations would not be sufficient justification for leaving the papers at home. However, she is also able to invoke an RM in support of her actions. From her point of view, this makes them defensible.

Whereas Cam in the previous extract invoked an RM in order to avoid having to do something, in Extract 6 patient Luong uses the same strategy to obtain something that she wants. She has pain in her back running down her legs.

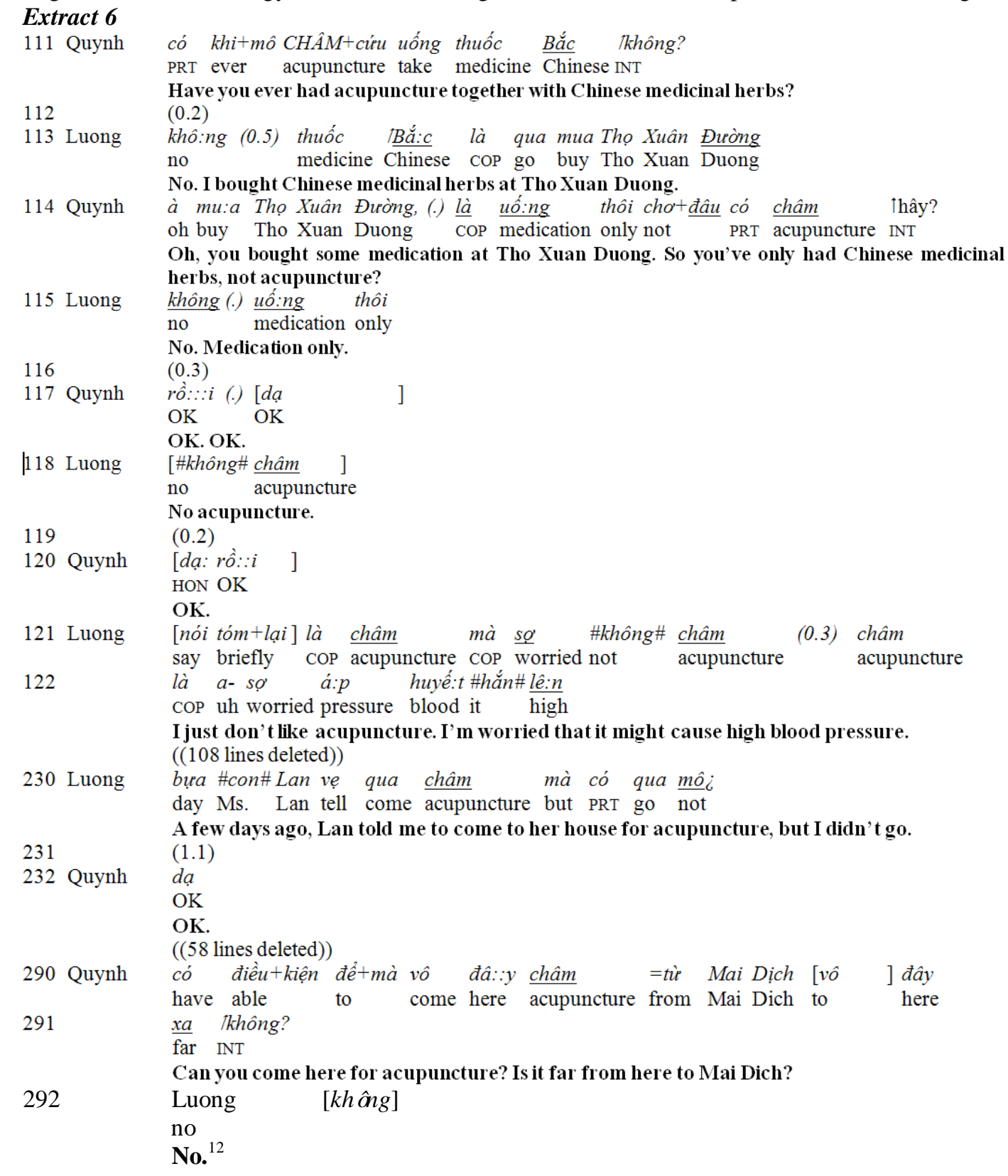

\footnotetext{
${ }^{12}$ This utterance is a response to only the first of Quynh's questions in lines 290-291.
} 


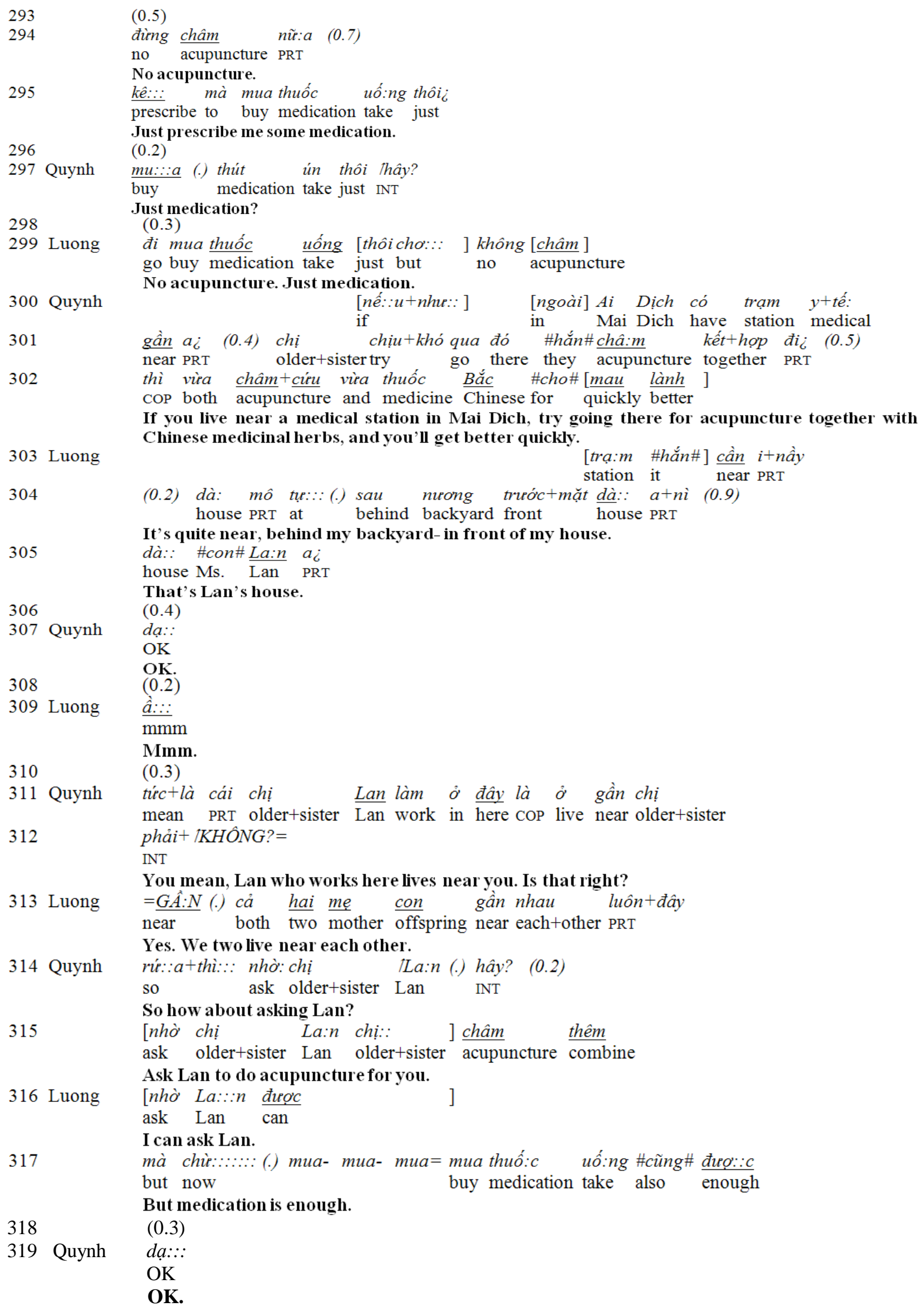

In the first sequence about Luong's previous treatment for her legs (lines 111-121), doctor Quynh asks if Luong's leg pain has been treated with acupuncture along with Chinese medicinal herbs (line 111). This elicitation displays Quynh's orientation towards her recommendation of acupuncture and Chinese medicinal herbs for Luong's problem. Anticipating Quynh's agenda, Luong rejects the option of acupuncture on the grounds that she thinks it may cause high 
blood pressure (lines 121-122). After 108 lines concerned with Luong's problems with her back and her blood pressure (data not shown), Luong rejects the acupuncture option again, this time invoking the third party, Lan, to support her rejection of this option (line 230). Luong's use of the recognitional form (i.e., Lan's personal name) implies that the referent is known to Quynh at this point. Quynh treats Luong's invocation of Lan as a form of resistance. In delayed fashion after 1.1 seconds (line 231), she shows her disaffiliation through a weak token, da ('OK; line 232). As the consultation develops, Quynh returns to her previous agenda by recommending acupuncture (in combination with Chinese medicinal herbs) for the third time (lines 290-291). Luong continues to actively resist the acupuncture treatment option (line 292). After two pauses (lines 293 and 295), Luong states the reason for her rejection of Quynh's treatment option, and requests an alternative, mua thuốc uống ('some medication'; line 296). Once again, Luong reaffirms her treatment preference (line 301) in her response to Quynh's request-for-confirmation question (line 299). Despite this resistance, Quynh sticks to her treatment recommendation (lines 301-303). In response, Luong mentions the location of the village hospital (lines 304-305 and 307), which triggers two utterances from Quynh: one (line 314) seeks Luong's confirmation, and one (line 315) acts as a recommendation. Luong responds to Quynh's recommendation using a pro forma agreement format (Schegloff, 2007), nhờ Lan đươc ('I can ask Lan'; line 316), as if to suggest that she will act on this recommendation. A pro forma agreement format means the speaker initially commits to the future course of action but later shows their disagreement. As it happens, Luong quickly reverts to her initial position (lines 317).

The exchange in Extract 6 indicates how patient Luong actively resists Quynh's treatment recommendation by refusing to take acupuncture. This is significant on two related levels. In general terms, it represents a triumph of the lay voice over the expert voice - a result which crucially depends on Lan's status as an RM. More particularly, this exchange also tells us something about the relative importance of collectivism and social status in determining treatment outcomes in this cultural context. As we saw above, Quynh's preferred treatment plan is that Luong should supplement her existing regime of medication with acupuncture. Luong's response is to associate the acupuncture option with another medical professional, Lan. This association then enables her to use her familial relationship with Lan to forestall Quynh's preferred treatment agenda and, ultimately, supplant it with her own. In short, as a determinant of her future treatment for her health problems, Luong's familial relationship with Lan takes precedence over Lan's status as a medical professional.

At the same time, Quynh's concession is problematic from a medical perspective (Stivers, 2006). In taking this step, she puts the final treatment decision in the hands of Luong on the basis that her health is her own responsibility: as a health provider, Quynh's role is only to give her advice, and not to impose her own treatment agenda on her. This step necessarily also has the effect of diminishing Quynh's accountability for any problems that might result from Luong's future treatment.

In Extract 6, Luong invoked an RM in order to obtain her preferred treatment option. The patient in Extract 7 also wants something, but this time it is a particular type of service. In her exchange with doctor Si, patient Nhu invokes her niece Tuyen, who is working in the same hospital, in an attempt to get the hospital to decoct some Chinese medicinal herbs for such an outpatient as her.

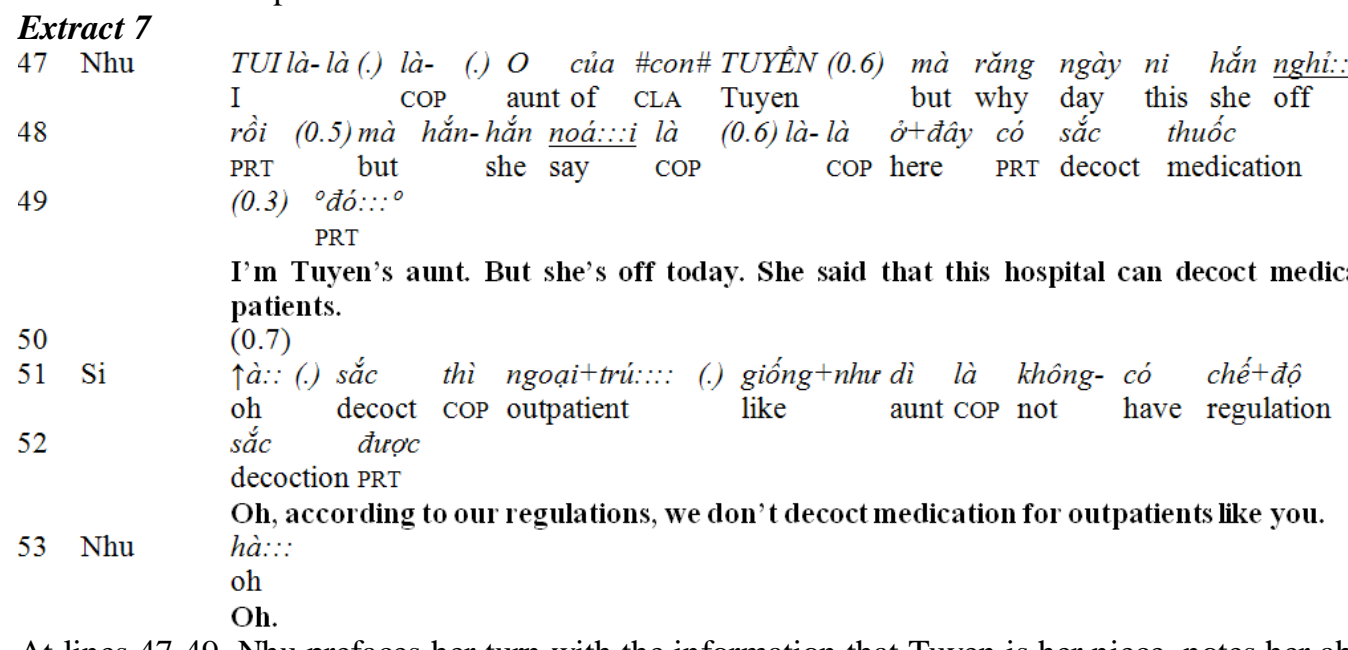

At lines 47-49, Nhu prefaces her turn with the information that Tuyen is her niece, notes her absence, and then raises the issue of medication decoction. She pauses several times between turn construction units to express her uncertainty about the veracity of the second and third pieces of information, while also stretching her talk at $\underline{n g h i \cdots}:$ ('off'), $\underline{\text { noá }:: i}$ ('she [i.e., Tuyen] said'), and ${ }^{o} \not o ́: .:^{o}$ to corroborate her claim. In response (lines 51-52), Si's upward-intoned $\uparrow a ̀$ :: ('oh'), plus details of the hospital regulations in second position, indicate that Nhu's sequence-initiating action has occasioned a marked shift of attention (Heritage, 1998). This aims to express a rejection of Nhu's presupposition. Nhu's stretched upshot, hà::: ('Oh'; line 53), then signals a change-of-state token (Heritage, 1984), and treats Si's information as new.

Nhu's first-pair part in this sequence (lines 47-49) is noteworthy in two respects. First, this turn is prefaced with information that seems irrelevant to the consultation: tui là $O$ của con Tuyền ('I'm Tuyen's aunt'). She raises her pitch 
on the name, TUYÊN, to draw attention to the fact that this is new information (Kidwell, 2013). In addition, Nhu's use of the final particle ${ }^{o}$ đó::: ${ }^{o}$ affords her claim the function of an announcement. Because she attributes the information about medication decoction to her niece, Nhu is able to disclose this information to Si in this forthright manner, which in turn legitimises her request that the hospital decoct medication for her.

So far in this section, we have seen patients invoke RMs in order to avoid something (Extract 5) or obtain something (Extracts 6 and 7). Neither of the remaining two extracts falls neatly into either of these categories. In each case, the patient explains why he has sought treatment in the current hospital rather than somewhere else. Consider Extract 8. Binh has suffered from seizures for four months, and was hospitalised for 20 days at a hospital in the city where the seizures started. Since he came to Vinh City three months earlier, Binh has not sought any treatment at all. This extract is concerned with doctor Yen's elicitation of information about his previous treatment.

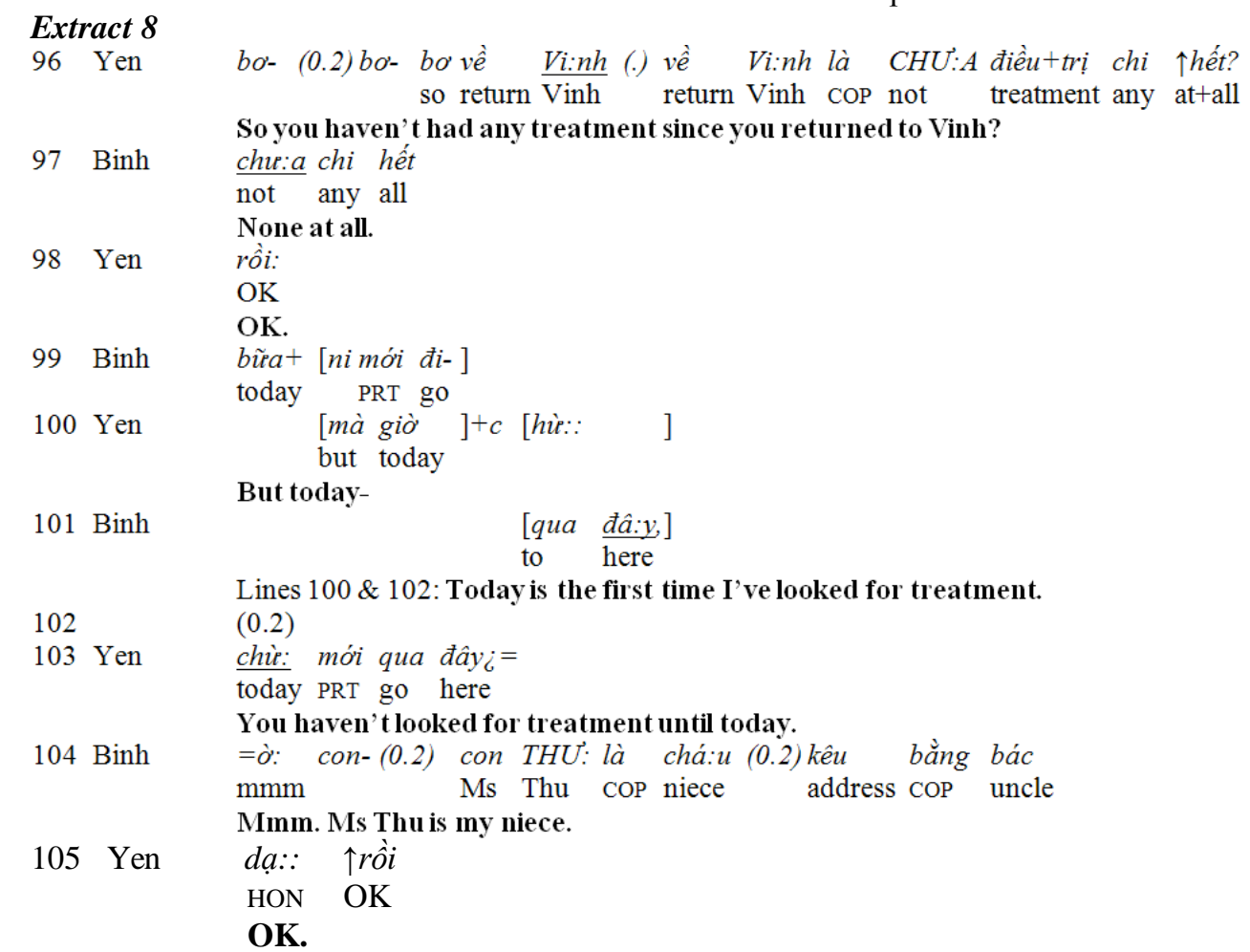

Doctor Yen opens the sequence with a question in the form of a declarative (lines 96-97). An utterance of this type formulates a 'B-event' (i.e., known to B, but not to A; Labov \& Fanshel, 1977), and counts as a request for information to which the patient has primary access. If it is negatively polarised, the use of a declarative question favours a 'no' response (Boyd \& Heritage, 2006), and this is what Binh supplies (line 98). Yen registers her receipt of Binh's information with rồi: ('OK'; line 99), without seeking any information. However, Binh adds further details to support his response (line 100). After several lines of post-start-up overlap (Gardner \& Mushin, 2007) and terminal overlapping talk (Jefferson, 1984), Yen resolves the overlap using a marked self-retrieval (Jefferson, 2004) at line 104. This resolution leads Binh to invoke an RM, his niece Thu, who also happens to work in this hospital (data not shown).

In this extract, Binh's invocation of an RM, Thu, accomplishes two possible social actions. To begin with, he justifies his decision to seek treatment at the current hospital. Binh returned to Vinh three months ago, but has not sought treatment anywhere for his seizures: this tells us that his seizures have not been serious enough to require urgent attention. In turn, we can conclude that Binh decided to seek treatment today on Thu's recommendation. Second, consider how Binh invokes his niece at line 105. He stutters at con- ('Ms.') and pauses for 0.2 seconds, before raising his pitch and stretching at the name of his niece, THU:. The stuttering and pause indicate that Binh is having difficulty disclosing the information that Thu is his niece. More to the point, however, the pitch-raising and stretching communicate Binh's desire to draw Yen's attention to his relationship with this RM in the hope that Yen will look after him especially conscientiously.

Whereas Binh leaves his reasons for choosing the current hospital implicit, Duc in Extract 9 makes them explicit to doctor Tung. In addition, as we shall see shortly, the situation depicted in this extract is more complex than the one in the previous extract in certain respects. This extract is taken after Duc presents his back problem.

\section{Extract 9}




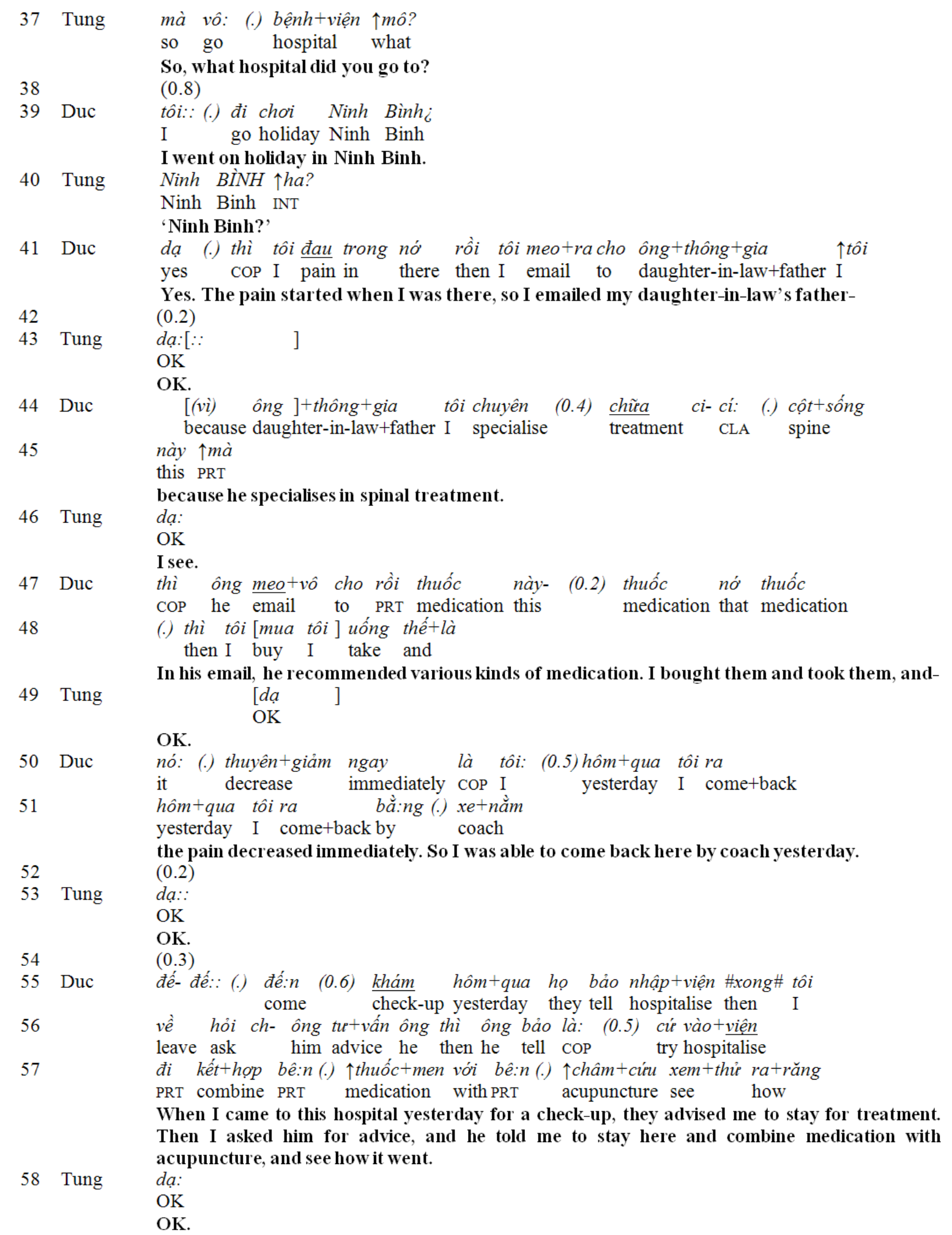

The talk included in this extract begins at the start of the consultation proper. As necessary background, note that Duc has just tried to take some medication in the presence of doctor Tung, but has been told not to. On witnessing this, Tung presupposes that Duc must have sought treatment elsewhere before coming to the present hospital (line 37). Duc responds with a narrative that spells out the reason why he chose this hospital (lines 39, 41, 44-45, 47-48, 50-51, and 55-57). In this narrative, he invokes his daughter-in-law's father, a specialist in spinal medicine (lines 44-45). Duc reveals that this RM has recommended some medication for him, and has advised him to seek treatment in this hospital (lines 47-48 and 55-57). However, this receives no assessment from Tung except an acknowledgement token (line 58).

Duc's invocation of an RM expresses an ambivalent attitude towards Tung. On one hand, by invoking someone who happens to be a clinical expert in the relevant medical field, Duc justifies purchasing medication for his back pain at another health centre before this consultation, and even attempting to take this medication right in front of his new 
attending doctor. On the other hand, Duc's invocation of the RM also provides him with a rationale for seeking treatment for a doctorable health issue in the present hospital, where, needless to say, he will be in the care of someone else again. In short, by opting to seek treatment here, Duc reveals a certain willingness to put his faith in Tung. It is also possible that, in invoking an RM in this situation, Duc hopes to receive better care from Tung than otherwise, as noted in our analysis of the consultation featured in Extract 8.

\section{CONCLUSION}

As we have seen, the participants in this study have diverse motives for invoking an RM in a medical consultation. Our data contains one instance in which this is done by the doctor. In Extract 4, Chu invokes an RM in order to elicit information from patient Sang, so our findings are in line with Bergmann's (1992) in this regard at least. At the same time, the information Chu elicits is not sensitive in nature, whereas in the earlier study the information elicited by the psychiatrists was indeed sensitive. This constitutes evidence that medical professionals invoke third parties as an elicitation strategy regardless of whether the information is sensitive or not. Needless to say, however, this conclusion is based on a limited pool of data. We need more work to be done before firm conclusions about the role played by informational sensitivity in this situation can be drawn.

Our analysis of the patient data has yielded three further results. First and foremost, the practice of invoking an RM in this cultural context empowers the patient to behave more assertively in a variety of medical scenarios than they might otherwise do (Fancher et al., 2010; G. T. Nguyen et al., 2007; K. Tran, 2009). One patient manages to circumvent a troublesome administrative requirement (Extract 5), while another invokes an RM in order to obtain a preferred form of treatment (Extract 6). In Extract 7, the patient wishes to receive a health-related service from the hospital, while also diminishing their accountability for making this request in case it turns out to be irregular. Still other patients adopt this practice in giving reasons for selecting the current hospital, and possibly also in the hope of receiving special attention from their treating doctor (Extracts 8 and 9). In Extract 9, the patient also invokes an RM in order to challenge the attending doctor's expertise.

Moreover, these motives for invoking third parties differ from the ones observed in earlier research conducted in the Western cultural context. ${ }^{13}$ Recall from Section 1 that the patient in Gill and Maynard (2006) invokes a third party in order to mitigate a frank and constraining question about her health condition. In Heritage and Robinson (2006), this practice serves to validate the patient's health concerns, and to diffuse their accountability for taking these concerns to the doctor as well. We ascribe this difference in observed motives to two interrelated properties of our study: the cultural context for the study, and the type of third party we have chosen for scrutiny. As foreshadowed in Section 1, our view is that a collectivist mentality plus a strongly-defined social hierarchy work in concert to promote the invocation of RMs in particular as third parties in Vietnamese medical communication.

Our third result is that, beyond merely promoting the use of this strategy, these two social forces appear to interact with each other in a particular way when an RM is invoked. In Extract 6, we saw that patient Luong's familial relationship with Lan overrode Lan's status as a medical professional in shaping the direction of her future treatment for her health problem. While data such as this is encouraging, the available evidence must be deemed only suggestive at this point. Further research on the putative effects of collectivism and social status on treatment outcomes is undoubtedly required.

\section{ACKNOWLEDGEMENTS}

The data for this study comes from the first author's doctoral research, which was supported by an Endeavour Scholarship and Fellowship from the Australian Government.

\section{REFERENCES}

[1] Appel, S. (2013). Vietnamese Americans. In J. Giger (Ed.), Transcultural nursing: Assessment and intervention (6th ed., pp. 426-461). St. Louis, MI: Elsevier.

[2] Bao Dat. (2001). The cultural aspects of communication reluctance in the EFL classroom: The case of Vietnamese students. English Teacher: An International Journal, 4(3), $232 . \quad$ Retrieved from http://www.aulibrary.au.edu/multim1/ABAC_Pub/The-English-Teacher-An-International-Journal/v4-n3-5.pdf.

[3] Bergmann, J. (1992). Veiled morality: Notes on discretion in psychiatry. In P. Drew \& J. Heritage (Eds.), Talk at work: Interaction in institutional settings (Vol. 8., pp. 137-162). Cambridge, England: Cambridge University Press.

[4] Boyd, E., \& Heritage, J. (2006). Taking the history: Questioning during comprehensive history-taking. In J. Heritage \& D. Maynard (Eds.), Communication in medical care: Interaction between primary care physicians and patients (pp. 151-184). Cambridge, England: Cambridge University Press.

[5] Drew, P. (1997). 'Open' class repair initiators in response to sequential sources of troubles in conversation. Journal of Pragmatics, 28(1), 69-101. doi:10.1016/S0378-2166(97)89759-7.

\footnotetext{
${ }^{13}$ While the patients in Extract 3 (from Heritage \& Robinson, 2006, p. 59) and Extract 7 both invoke third parties in order to diffuse accountability for their actions, they do this for different reasons.
} 
[6] Drew, P. (2013). Turn design. In J. Sidnell \& T. Stivers (Eds.), The handbook of conversation analysis (pp. 131-149). Malden, MA: Wiley-Blackwell.

[7] Edwards, V., \& Phan, A. (2013). Managers and management in Vietnam: 25 years of economic renovation (Doi moi). New York, NY: Routledge.

[8] Enfield, N. J., Stivers, T., \& Levinson, S. C. (2010). Question-response sequences in conversation across ten languages: An introduction. Journal of Pragmatics, 42(10), 2615-2619. doi:10.1016/j.pragma.2010.04.001.

[9] Fancher, T., Ton, H., Le Meyer, O., Ho, T., \& Paterniti, D. (2010). Discussing depression with Vietnamese American patients. Journal of Immigrant and Minority Health, 12(2), 263-266. doi:10.1007/s10903-009-9234-y.

[10] Gardner, R., \& Mushin, I. (2007). Post-start-up overlap and disattentiveness in talk in a Garrwa community. Australian Review of Applied Linguistics, 30(3), 35.1-35.14. doi:10.1075/aral.30.3.06gar.

[11] Gill, V. T. (1998). Doing attributions in medical interaction: Patients' explanations for illness and doctors' responses. Social Psychology Quarterly, 61, 342-360. doi:10.2307/2787034.

[12] Gill, V. T., \& Maynard, D. (2006). Explaining illness: Patients' proposals and physicians' responses. In J. Heritage \& D. Maynard (Eds.), Communication in medical care: Interaction between primary care physicians and patients (pp. 115-150). Cambridge, England: Cambridge University Press.

[13] Gill, V. T., \& Roberts, F. (2013). Conversation analysis in medicine. In J. Sidnell \& T. Stivers (Eds.), The handbook of conversation analysis (pp. 575-592). Chichester, England: Wiley-Blackwell.

[14] Haakana, M. (2001). Laughter as a patient's resource: Dealing with delicate as pects of medical interaction. Text, 21(1-2), 187219. doi:10.1515/text.1.21.1-2.187.

[15] Heritage, J. (1984). A change-of-state token and aspects of its sequential placement. In J. M. Atkinson \& J. Heritage (Eds.), Structures of social action: Studies in conversation analysis (pp. 299-345). Cambridge, England: Cambridge University Press.

[16] Heritage, J. (1998). Conversation analysis and institutional talk: Analyzing distinctive turn-taking systems. In S. Cmejrková, J. Hoffmannová, \& O. Müllerová (Eds.), Dialoganalyse VI (Volume 2) (Proceedings of the 6th International Congress of IADA International Association for Dialog Analysis) (pp. 3-17). Tübingen, Germany: Niemeyer.

[17] Heritage, J., \& Robinson, J. D. (2006). Accounting for the visit: Giving reasons for seeking medical care. In J. Heritage \& D. Maynard (Eds.), Communication in medical care: Interaction between primary care physicians and patients (pp. 48-85). Cambridge, England: Cambridge University Press.

[18] Hofstede, G. (2001). Culture's consequences: Comparing values, behaviours, institutions, and organizations across nations. Thousand Oaks, CA: Sage Publications.

[19] Jefferson, G. (1984). Notes on some orderlinesses of overlap onset. In V. D’Urso \& P. Leonardi (Eds.), Discourse analysis and natural rhetoric (pp. 11-38). Padua, Italy: Cleup Editore.

[20] Jefferson, G. (2004). Glossary of transcript symbols with an introduction. In G. Lerner (Ed.), Conversation Analysis: Studies from the first generation (pp. 13-31). Philadelphia, PA: John Benjamins.

[21] Kádár, D., \& Mills, S. (2011). Politeness in East Asia. Cambridge, England: Cambridge University Press.

[22] Kidwell, M. (2013). Interaction among children. In J. Sidnell \& T. Stivers (Eds.), The handbook of conversation analysis (pp. 511-532). Chichester, England: Wiley-Blackwell.

[23] Kim, M.-S., Klingle, R., Sharkey, W., Park, H.-S., Smith, D., \& Cai, D. (2000). A test of a cultural model of patients' motivation for verbal communication in patient-doctor interactions. Communication Monographs, 67(3), $262-283$. doi:10.1080/03637750009376510.

[24] Labov, W., \& Fanshel, D. (1977). Therapeutic discourse: Psychotherapy as conversation. New York, NY: Academic Press.

[25] Nguyen, H. D. (2009). Vietnamese. In Comrie, B. (Ed). The world's major languages (2nd ed.; pp. 677-692). London, England: Routledge.

[26] Nguyen, G., Barg, F., Armstrong, K., Holmes, J., \& Hornik, R. (2007). Cancer and communication in the health care setting: Experiences of older Vietnamese immigrants, a qualitative study. Journal of General Internal Medicine, 23(1), 45-50. doi:10.1007/s11606-007-0455-2.

[27] Nguyen, H. T. L., Austin, G., Chau, D. D., Nguyen, H. Q., Nguyen, K. H. B., \& Duong, M. T. (2018). Eliciting patients' health concerns in consulting rooms and wards in Vietnamese public hospitals. International Journal of Applied Linguistics \& English Literature, 7(2), 121-133.

[28] Nguyen, T. X. (1994). The Vietnamese family moral code. In T. X. Nguyen (Ed.), Vietnamese studies in a multicultural world (pp. 73-80). Melbourne, Australia: Vietnamese Language \& Culture Publications.

[29] Pham, N. T. H. (2014). Linguistic and cultural constraints in Vietnamese general practitioners' act of initiating clinical information-seeking process in first encounters with outpatients. Theory and Practice in Language Studies, 4(6), $1125-1131$. Retrieved from http://www.academypublication.com/issues/past/tpls/vol04/06/04.pdf.

[30] Pomerantz, A. (1980). Telling my side: "Limited access" as a "fishing" device. Sociological Inquiry, 50(3-4), 186-198. doi:10.1111/j.1475-682X.1980.tb00020.x.

[31] Pomerantz, A. (1984). Agreeing and disagreeing with assessments: Some features of preferred /dispreferred turn shaped. In J. M. Atkinson \& J. Heritage (Eds.), Structures of social action: Studies in conversation analysis (pp. 57-101). Cambridge, England: Cambridge University Press.

[32] Raymond, G. (2003). Grammar and social organization: Yes/no interrogatives and the structure of responding. American Sociological Review, 68(6), 939-967. doi:10.2307/1519752.

[33] Schegloff, E. (2007). Sequence organization in interaction: A primer in conversation analysis I. Cambridge, England: Cambridge University Press.

[34] Schegloff, E., Jefferson, G., \& Sacks, H. (1977). The preference for self-correction in the organization of repair in conversation. Language, 53(2), 361-382. doi:10.1353/lan.1977.0041.

[35] Sidnell, J. (2012). "Who knows best?": Evidentiality and epistemic asymmetry in conversation. Pragmatics \& Society, 3(2), 294-320. doi:10.1075/ps.3.2.08sid. 
[36] Stivers, T. (2006). Treatment decisions: Negotiations between doctors and parents in acute care encounters. In J. Heritage \& D. Maynard (Eds.), Communication in medical care: Interaction between primary care physicians and patients (pp. 279-312). Cambridge, England: Cambridge University Press.

[37] Thompson, L. (1965). A Vietnamese grammar. Seattle, WA: University of Washington Press.

[38] Tran, V. Q., To, T. N., Nguyen, B. C., Lam, D. M., \& Tran, A. T. (1998). Cơ sở văn hóa Việt Nam [Vietnam's cultural basis] (8th ed.). Hanoi, Vietnam: Nhà xuất bản giáo dục Việt Nam [Viet Nam Education Publishing House].

[39] Tran, K. (2009). Examining conversational constraints in Vietnamese patient-doctor communication: A case study. XULAneXUS: Xavier University of Louisiana's Undergraduate Research Journal, 6(1), 3-20. Retrieved from http://xulanexus.xula.edu/textpattern/index.php?s=file_download\&id=20.

Huong Thi Linh Nguyen obtained her bachelor's degree in 2003 and an MA in English education in 2011, both at Hue University, Vietnam. She is currently a PhD candidate in applied linguistics at the University of Southern Queensland, Australia, supported by an Endeavour Scholarship and Fellowship. Her research interests are medical communication, Conversation Analysis and learner autonomy.

Gavin Austin is a lecturer in applied linguistics at the University of Southern Queensland, Australia. His main area of teaching and research is second language acquisition, though he also has expertise in linguistic theory, quantitative research methods and discourse analysis. In addition to lecturing at tertiary level, Gavin has worked as an English language instructor in Japan and Australia. 